\title{
El impacto de la educación alimentaria nutricional en el embarazo: una revisión de las experiencias de intervención
}

\author{
Impact of food and nutrition education \\ during pregnancy: a review \\ of nutritional interventions
}

\begin{abstract}
Pregnancy is a period of great nutritional vulnerability in a women's life, thus food and nutrition education (FNE) is a valuable tool for achieving healthy eating. The purpose of this study was to review the impact of nutritional interventions designed for healthy pregnant women. An analysis of the geographical distribution of 42 publications and the educational strategies employed was conducted. Effectiveness to generate changes in knowledge, eating attitudes and practices, promote an adequate weight gain and to prevent anemia was analyzed. Research from Asia and those including a combination of multiple educational strategies such as written information, information and communication technologies, workshops or discussion groups were the most prevalent. Interventions show favorable results when they intend to achieve an increase in the level of knowledge, stimulate changes in dietary patterns and prevent iron-deficiency anemia by adherence to iron supplementation. Gestational weight gain was also favorably affected when FNE is employed, however, the prevention of excessive weight gain depended on nutritional status prior to pregnancy and on other genetic and environmental determinants. Successful experiences encourage the inclusion of FNE as a routine tool in prenatal care; personalized nutritional counselling and the use of ICT are alternatives of choice in the current health scenario.
\end{abstract}

Key words: Pregnancy; nutrition during pregnancy; prenatal care; health education; nutritional intervention.

\section{INTRODUCCIÓN}

El embarazo es uno de los momentos de mayor vulnerabilidad nutricional en la vida de la mujer ya que las necesidades de energía y nutrientes se encuentran aumentadas en virtud del crecimiento fetal y de la síntesis de tejidos maternos como el aumento del tamaño uterino, la hipertrofia de las glándulas mamarias y la acumulación de las reservas grasas. El estado nutricional de la madre previo a la gestación y los hábitos alimentarios implementados durante el embarazo, son condicionantes de importancia en este periodo del ciclo vital (1-3)

Mantener una alimentación saludable es un objetivo primordial del cuidado prenatal, momento en el cual la edu-
Bárbara Puszko

Sofía Sánchez

Noelia Vilas

Melisa Pérez

Luciana Barretto

Laura López

Escuela de Nutrición. Facultad de Medicina Universidad de Buenos Aires. Argentina

Dirigir la correspondencia a: Profesora Laura Beatriz López Marcelo T. de Alvear 2202, 4to piso, CP: 1120

CABA. Argentina

Email: Iblopez@fmed.uba.ar/Iblopez@yahoo.com.ar

Este trabajo fue recibido el 18 de Julio de 2016 y aceptado para ser publicado el 22 de Diciembre de 2016.

cación alimentaria nutricional (EAN) resulta una herramienta imprescindible que permite reforzar los comportamientos alimentarios beneficiosos y modificar aquellos que impactan negativamente sobre la salud. Alcanzar dichas modificaciones requiere que la mujer tenga un papel activo, sea consciente de los riesgos de llevar a cabo una alimentación inadecuada y testigo de sus propios avances y logros (4).

La ganancia de peso y la deficiencia de algunos micronutrientes críticos constituyen las principales problemáticas a ser abordadas en el control prenatal mediante la EAN. Por la relación que existe entre el peso materno y el desarrollo y crecimiento fetal, es importante controlar que la ganancia de peso gestacional se encuentre dentro de un rango saludable (5, 
6). La malnutrición por exceso incrementa el riesgo de partos por cesárea, diabetes mellitus, macrosomía fetal, defectos del tubo neural, como así también enfermedades crónicas no transmisibles en la vida adulta. Mientras que un aumento de peso insuficiente es un factor de riesgo de aborto espontáneo, parto prematuro y bajo peso al nacer $(7,8)$.

En relación con la deficiencia de micronutrientes, según los datos del último reporte de la Organización Mundial de la Salud (OMS) publicado en 2015, se estima que el 38,2\% $(95 \% \mathrm{Cl}: 33,5-42,6)$ de las embarazadas en el mundo son anémicas, siendo la carencia de hierro la principal causa (9). La anemia durante el embarazo se asocia directamente con un elevado riesgo de parto prematuro, bajo peso al nacer y mortalidad materna y neonatal. Su prevención es crítica y tradicionalmente se ha logrado a través de la suplementación y fortificación de alimentos (10).

Es reconocido que durante la gestación, las mujeres están predispuestas a recibir información que les resulte útil para llevar adelante un embarazo saludable, a su vez, los profesionales de la salud encuentran más oportunidades para asistirlas. Por este motivo es fundamental jerarquizar en esta etapa del ciclo vital el papel de las intervenciones nutricionales, especialmente las de carácter educativo, cuyo fin último sea lograr la adquisición de los conocimientos necesarios por parte de la gestante y la confianza suficiente para aplicarlos durante todo el desarrollo del embarazo. Por estos motivos, algunos autores han descripto al embarazo como un momento muy propicio para la enseñanza, en el cual las mujeres tienen consciencia sobre su cuerpo y sobre su responsabilidad ante una nueva vida (11).

En este contexto, el diseño de intervenciones nutricionales basadas en la EAN que logren estimular hábitos saludables durante el embarazo es un desafío actual para los equipos de salud, por lo que este estudio tuvo el objetivo de realizar una revisión de los impactos que han tenido experiencias de EAN destinadas a mujeres embarazadas en distintos ámbitos geográficos.

\section{MÉTODOS Y FUENTES DE INFORMACION}

Se consideraron elegibles las investigaciones publicadas en el periodo comprendido entre los años 2000 y 2015. Los motores de búsqueda de información fueron: PubMed (National Library of Medicine, Estados Unidos), LILACS (Literatura Latinoamericana de Información en Ciencias de la Salud) y SCIELO (Scientific Electronic Library Online). Se utilizaron los descriptores: embarazada, consejería nutricional, intervención nutricional, cuidados prenatales, educación nutricional, suplementación, suplemento, prevención de anemia, ganancia de peso gestacional, educación saludable, promoción de comportamientos saludables, comunicación saludable, así como sus respectivas traducciones al idioma inglés.

Las publicaciones fueron seleccionadas cuando incluían una intervención nutricional con componente educativo propuesto para embarazadas adultas, incluyendo estudios destinados a evaluar gestantes que presentaron anemia y aquellas intervenciones que tenían como destinatarias a embarazadas independientemente de su estado nutricional. Se excluyeron las investigaciones que estaban orientadas específicamente a gestantes con patologías como diabetes gestacional, hipertensión arterial, obesidad, entre otras. De las 152 investigaciones identificadas por los términos de búsqueda, 42 fueron elegidas para conformar el presente trabajo. Se realizó una descripción de la distribución geográfica de las investigaciones y de las estrategias educativas empleadas, analizándose luego la efecti- vidad para generar cambios en los conocimientos, actitudes y prácticas alimentarias, ganancia adecuada de peso y utilización de suplementos.

\section{RESULTADOS}

En la tabla 1 se presentan las características generales de los estudios incluidos en la revisión. Las unidades de análisis según el ámbito geográfico de procedencia muestran un predominio de investigaciones provenientes de Asia (12-23) seguidas por las experiencias de América del Norte (24-34), Europa (35-42), América Latina (43-49) y en menor medida las procedentes de Oceanía (50-51) y África (52-53).

\section{Características de las Estrategias educativas empleadas}

Para alcanzar los objetivos de investigación propuestos en los trabajos incluidos, los educadores seleccionaron experiencias de aprendizaje de distinta naturaleza. La mayoría de los estudios incluidos en la revisión (81\%) utilizaron una combinación de múltiples estrategias, incluyendo en forma conjunta información escrita, el uso de tecnologías de información y comunicación (TICS) u otras formas como talleres o grupos de discusión.

La información escrita fue el modelo más empleado, predominando en esta modalidad la trasmisión de pautas higiénico - dietéticas y la realización de planes de alimentación personalizados. En menor medida se utilizaron como estrategias el uso de folletería, la entrega de libretas u otros instrumentos para auto registro, listas de alimentos para guiar la compra, tarjetas para la adquisición o canje de alimentos y la entrega de recetas.

Las TICs fueron utilizadas en segundo lugar como estrategias de intervención en los estudios seleccionados. En la actualidad internet y la telefonía celular se han convertido en un elemento común y casi indispensable en la vida cotidiana de las personas. Estos instrumentos modificaron la manera en que la población vive e interactúa, estimulando a las autoridades sanitarias y a los profesionales de la salud a valerse de tecnologías de la información como herramientas de prestación de servicios. Según la OMS se entiende por cibersalud al uso eficaz de las TICs para fomentar la salud, este organismo junto con la Unión Internacional de Telecomunicaciones ha destacado el papel fundamental que desempeña esta herramienta para alcanzar los Objetivos de Desarrollo del Milenio en las poblaciones más vulnerables del mundo $(54,55)$. En los trabajos incluidos en la revisión, el envío de información vía mail (pautas higiénico dietéticas, postales electrónicas, gráficas personalizadas de incremento de peso y recetas), fue una de las modalidades más implementadas. Se describieron también el uso de diferentes herramientas para auto monitoreo, las que favorecen la retroalimentación y la consulta con el profesional. Con menor frecuencia se emplearon videos, llamadas telefónicas, difusión de mensajes a través de televisión y radio, uso de diapositivas en sesiones grupales y mensajería de texto.

Otras estrategias educativas empleadas incluyeron la trasmisión de información oral junto con la entrega de suplementos, el desarrollo de talleres con demostraciones y grupos de discusión y la entrega de complementos alimentarios.

En relación al número de personas intervinientes en la modalidad educativa, la mayoría de las experiencias emplearon sesiones individuales, sólo en el $12 \%$ se llevaron a cabo intervenciones de intercambio como talleres o grupos y el 28 $\%$ utilizó ambos tipos de modalidades.

En los estudios en los que se especifica que profesionales estuvieron a cargo de realizar las intervenciones educativas, se 
TABLA 1

Características de los estudios incluidos en la revisión.

\section{Características de las experiencias de intervención}

Lugar Geográfico del estudio

Asia

EEUU y Canadá

Europa

América Latina

Oceanía

África

Objetivos de la intervención educativa utilizada

Producir cambios en los conocimientos y/o prácticas alimentarias.

Estimular una adecuada la ganancia de peso

Prevenir la anemia mediante el uso de suplementos junto

con actividades educativas

Formato educativo empleado

Trasmisión escrita

Pautas higiénico dietéticas

Planes de alimentación personalizados

Entrega de Folletos

Instrumentos para autoregistro

Guías para la compra de alimentos

Tarjetas para la compra de alimentos

Recetarios

Uso de TICs

Correo electrónicos

Aplicaciones para auto monitoreo

Videos

Llamadas Telefónicas

Televisión y radio

Filminas

Mensajería de textos

Otras formas

nformación oral

Talleres, grupos de debate o discusión, entrega de suplementos

Modalidad de participación empleada

Individual

Grupal

Ambas

Periodo del embarazo en el cual se incluye la intervención

Desde el inicio del embarazo hasta la semana 16 de gestación

Hasta la semana 26 de gestación

Periodo de intervención no definido

No se especifica

Profesionales responsables de la Educación

Médicos, Obstétricas, Enfermeros

Nutricionistas

No se especifica
Cantidad

de estudios

Referencias

$12-23$

24-34

35-42

43-49

50,51

52,53

$12-17,24-27,35-39,43-46,50-52$

$17-19,24,26,28-34,36,38,40-42,46,47$

$14-17,20-23,45,46,48,49,53$

23

13

10

6

4

2

2

4

3

2

2

2

1

22

8

25

5

12

16

20

4
2
$12,14,17,19,21,24-26,28,29,31-34,36,38$ $41,46,48,50,53$

$18,25,26,29,31,32,34,35,36,40,41,48,51$

$13,17-19,22,27,36,42,45,50$

$15,27,33,36,41,46$

$27,37,38,48$

27,50

38,43

$27,31-33,36,38$

$18,25,30,33$

$24,25,43$

31,35

43,53

19,51

23

$13,15,16,18,19,20,22,25,26,28-$

$30,35,36,40,42-45,47,49,52$

$13,20,27,30,37,43,46,52$

$14,17,18,22-24,27,28,31-33,35-42,44,47-51$

$13,20,21,30,46$

$12,15,16,19,25,26,29,34,43,45,52,53$

$12,15,18,23,28,31,35,36,39,40,42,45,46$ $48,51,52$

$14,16,17,19,21,22,24-27,29,30,32$

$34,37,38,41,47,49$

$13,20,50,53$

43,44

$17-19,21,22,24,32,33,41-43,46,47,49,51,53$

$12,25,26,28,29,35,37-40,45$

$13-16,20,23,27,30,31,34,36,44,48,50,52$ 
TABLA 2

Estudios que tuvieron como objetivos mejorar los conocimientos, actitudes y/o prácticas alimentarias durante el embarazo mediante intervenciones nutricionales (Gl: grupo intervención; GC: grupo control).

\section{Autor, Año}

Ahrari M. y cols. 2006 (52)

Al-Tell M. A. y cols. 2010 (15)

Bosaeus M. y cols. 2015 (35)

País

Egipto

Palestina

Suecia

Briley C. y cols. 2002 (25)

Emmett R. y cols. 2015 (50)

Fallah F. y cols. 2013 (12)

Garg A. y cols. 2006 (16)

Gutierrez Maydata A. y col. 2006 (43)

Cuba

Hui A.y cols. 2014 (26)

Jackson R. y cols. 2011 (24)

Khoramabadi M. y cols. 2015 (13)

Kinnunen T. y cols. 2007 (36)

Laitinen K. y cols. 2009 (37)

McGowan C. y cols. 2013 (38)

Moreno Mejía C. y col. 2011 (44)

Noronha J. y cols. 2013 (14)

Oken E. y cols. 2013 (27)

Parra B. E. y col 2005 (45)

Piirainen T. y cols. 2006 (39)

Restrepo S. y col. 2010 (46)

Shivalli S. y cols. 2015 (17)

Wilkinson S. y cols. 2012 (51)

\section{Principales Hallazgos}

El GI reportó una mayor ingesta de alimentos ( $54,9 \%$ vs $10,6 \%)$, carne $(57,1 \%$ vs $4,2 \%)$ y vegetales $(66,9 \%$ vs $5,3 \%)$ comparado con el GC.

El GI demostró una mejora significativa en sus hábitos y prácticas alimentarias en comparación con el GC $(p<0,05)$.

El consumo semanal de pescado aumentó en el GI, desde el primer al segundo trimestre $(p=0,03)$ y desde el segundo al tercer trimestre $(p=0,01)$. En el primer trimestre el consumo de pescado se correlacionó con los valores serológicos de EPA $(r=0,36, p=0,002)$ y de DHA $(r=0,34, p=0,005)$ y el consumo de carne se correlacionó con los valores serológicos de ARA $(r=0,28, p=0,02)$.

E.E.U.U. $\quad$ El Gl incrementó el consumo de hierro $(p<0,01)$. La vitamina B6 y folato tendieron a ser más elevados $(p<0,10)$ luego de la intervención.

Australia Hubo un aumento significativo en el conocimiento de alimentos fuente de DHA $(p<0,001)$ y en el consumo de pescado $(p<0,001)$.

Irán El nivel de conocimientos sobre alimentación saludable perteneciente a la categoría "bueno", se incrementó del $3 \%$ al $31 \%$ luego de la intervención y el conocimiento calificado como "débil" disminuyó del 31\% al $6 \%(\mathrm{p}<0,01)$.

India Hubo un aumento significativo en la cantidad de casi todos los grupos de alimentos consumidos en $\mathrm{Gl}$ en comparación con el GC (cereales, frutas, hortalizas, leche, carnes; $\mathrm{p}<0,001)$. El Gl tuvo un aporte mayor de kcal, proteínas, calcio, hierro, Vitamina $\mathrm{C}$ y $\mathrm{A}$ en comparación con el GC $(p<0,001)$. Sin embargo, esta mejora alcanzo el nivel de adecuación nutricional solo en la grasa, vitamina $A$ y la ingesta de vitamina $C$.

Hubo modificaciones favorables, tanto en el conocimiento como en las motivaciones, preferencias y destrezas en la preparación de las dietas, con un mayor consumo de antioxidantes $(p<0,01)$

El GI tuvo una menor ingesta calórica, de grasas totales, grasas saturadas y colesterol a los 2 meses de haber iniciado el estudio en comparación con el GC $(p<0,001)$

E.E.U.U. $\quad$ En el GI hubo una mejoría en la alimentación (aumento del consumo de frutas, verduras, cereales integrales, pescados y frutas secas y una disminución del consumo de alimentos azucarados, cereales refinados, alimentos fritos, comida rápida y grasas solidas; $p<0,05)$ y en los conocimientos nutricionales $(p=0,0001)$.

Irán El Gl presentó un mayor nivel de conocimiento, mejora de en los hábitos alimentarios y percepción del riesgo y de barreras comparado con el GC $(p<0,05)$

Finlandia La ingesta de verduras y frutas así como la de fibra dietética fue mayor en el Gl comparado con el GC ( $p=0,004 ; p=0,007)$.

Finlandia En el grupo que recibió asesoramiento nutricional con probióticos se evidenció una menor glucemia $(p=0,025)$, mejor tolerancia a la glucosa $(p=0,013)$, así como también una menor concentración de insulina $(p=0,032)$.

Irlanda El Gl tuvo una ingesta significativamente menor de energía $(p<0,05)$, mayor de proteínas $p<0,01)$, fibra dietética $(p<0,01)$, vitamina $A(p<0,05)$ y Magnesio $(p<0,001)$. El consumo de alimentos con alto índice glucémico fue reducido significativamente en el $\mathrm{Gl}$.

Perú La mayoría de las participantes mejoraron en el postest, alcanzando un nivel de conocimiento bueno en un $73,3 \%$ y regular en $26,7 \%(p<0,05)$

India El puntaje de ganancia media de conocimiento del $\mathrm{Gl}$ fueron más altos que en los $\mathrm{GC}(\mathrm{p}<0,01)$. Asimismo se observó un mejor puntaje en la habilidad para seleccionar alimentos en el GI con respecto a los controles $(p<0,01)$

Estados Unidos En los grupos que recibieron asesoramiento y asesoramiento con tarjeta de compra se evidenció un incremento significativo en el consumo de pescado $(p=0,01)$ y de DHA $(p<0,0001)$. No se hallaron diferencias significativas en relación a la ingesta de Mercurio o en los biomarcadores.

Colombia Existieron cambios significativos en los conocimientos sobre las prácticas que se deben implementar para mejorar la biodisponibilidad del hierro, disminuir pérdidas de folatos, así como también se ampliaron los conocimientos sobre fuentes de hierro, folatos y vitamina $C .(p<0,003)$.

Finlandia Las mujeres del GI presentaron ingestas más elevadas de verduras, frutas, aceites vegetales y menor consumo de manteca que las del GC. La ingesta de vitamina E, ácido ascórbico y folato fue mayor en el $\mathrm{Gl}$, no se hallaron diferencias en el consumo de otros nutrientes. Se incrementó en promedio el consumo de 300 kcal por día $(p<0,001)$. Antes de iniciar el programa de intervención, 91\% de las participantes presentaban riesgo de deficiencia en la ingesta usual de calorías, deficiencia que se redujo a $81,6 \%$ en la evaluación realizada a las madres en el tercer trimestre. Se presentó un incremento estadísticamente significativo en el consumo de hierro, vitamina $A, C$ y folatos $(p<0,001)$.

India La ingesta proteica promedio fue mayor en el $\mathrm{Gl}$ que en el GC $(p<0,05)$. Más de dos tercios de las mujeres del $\mathrm{Gl}$ incorporaron una comida extra y sólo un tercio de los controles.

Australia Las mujeres que participaron del taller aumentaron su consumo de porciones de frutas $(p=0,004)$, verduras $(p=0,006)$, obtuvieron un puntaje más alto de calidad de la dieta $(p=0,027)$ y mejoraron sus conocimientos sobre la ganancia de peso óptima en el embarazo. 
mencionan con mayor frecuencia a los médicos, obstétricas, obstetras, enfermeros y ginecólogos como los responsables de las mismas. Los nutricionistas participaron, ya sea en forma individual o interdisciplinaria, en el $37 \%$ de los estudios.

Efectividad de la educación alimentaria para producir cambios en los conocimientos y prácticas alimentarias.

Entre las intervenciones destinadas a modificar el comportamiento alimentario de las gestantes mediante la aplicación de EAN, 8 de los 22 estudios seleccionados se propusieron mejorar el nivel de conocimientos de las embarazadas en relación a la alimentación. La eficacia de la intervención se evaluó mediante la aplicación de un cuestionario previo y otro posterior a la misma y en todos los casos se observó una mejora en el nivel de conocimiento alcanzado (12-14, 24, $43-45,50)$. Entre las actividades de aprendizajes utilizadas para lograr los cambios observados, se destacan la utilización de sesiones individuales o grupos pequeños $(12,44,45)$, el uso de video debate, la confección de recetas y la entrega de folletos y tarjetas de compra $(43,50)$.

Es conocido que la posibilidad de lograr cambios en los hábitos alimentarios está en estrecha relación con conocer y modificar la causa que los ha generado, siendo éste un proceso complejo y difícil de alcanzar, especialmente en aquellos que están sustentados por la religión o responden a la conservación de costumbres (56). En quince de las investigaciones analizadas, la necesidad de implementar prácticas saludables en las embarazadas promovió el desarrollo de intervenciones tendientes a mejorar la ingesta de nutrientes críticos y/o el aporte energético diario. La totalidad de los estudios realizados en este sentido lograron cambios positivos en la calidad de la alimentación o en el consumo de determinados alimentos respondiendo a objetivos particulares como un incremento del consumo de frutas y verduras, leche, cereales integrales, carnes magras y aceites de buena calidad (15-17, 24, 26, 27, $35,36,38,39,43,46,50-52)$.

Dada la relevancia que el aporte dietético de ácido docosahexaenoico (DHA) presenta durante el embarazo, fue el propósito de otras tres investigaciones mejorar la ingesta de este nutriente promoviendo el consumo seguro de pescado, objetivo que en todos los casos se logró mediante la intervención nutricional $(27,35,50)$. Por otro lado, en el estudio realizado por Oken E. y cols. no observaron diferencias significativas en la concentraciones de mercurio o de sus biomarcadores entre los grupos de intervención y control (27).

Cuatro autores reportaron luego de la intervención, un incremento en la ingesta de micronutrientes, destacándose el hierro, calcio, magnesio, ácido fólico, vitaminas $A, C$ y del complejo $B(16,25,38,46)$. Asimismo se pondera el incremento del consumo proteico $(16,17,38)$ y la adecuación del aporte calórico $(16,26,38,46)$.

Uno de los trabajos tuvo como meta una intervención para prevenir las complicaciones que pueden generar los valores alterados de glucemia durante la gestación y reporta cambios favorables en las concentraciones de glucosa e insulina plasmática luego de modificar la alimentación de las embarazadas implementando planes alimentarios de bajo índice glucémico (37). La tabla 2 resume los principales hallazgos de estos estudios.

\section{Efecto de la educación alimentaria en la ganancia de peso gestacional}

El impacto de un adecuado aumento de peso durante el embarazo es importante tanto para la salud materna como fetal, por lo que diversos autores plantearon intervenciones de EAN con la finalidad de estimular una ganancia de peso gestacional dentro las los rangos actualmente recomendados. La mayoría de los estudios cotejan la ganancia ponderal con los incrementos propuestos por el Instituto de Medicina de los Estados Unidos, basado en el Índice de Masa Corporal (IMC) pregestacional y según el año de publicación, se guiaron con la versión del año 1990 o 2009 (57, 58). Dos de los estudios utilizaron otros estándares de evaluación de carácter nacional o regional como la propuesta del Departamento de Salud de Taiwán y la gráfica proveniente de Chile $(18,46)$.

El propósito particular de las intervenciones estuvo direccionado según las características epidemiológicas de la población objetivo y tuvo la finalidad ya sea de estimular o bien de limitar el aumento de peso; cuya evaluación fue realizada en algunos casos considerando una dimensión continua de la variable o bien en forma categórica en base a un patrón normativo. La tabla 3, presenta un resumen de estas investigaciones.

Dieciséis de las investigaciones analizadas se propusieron limitar la ganancia de peso excesiva durante la gestación (18, $24,26,28-34,36,38,40-42,47)$, seis de ellas demostraron un efecto favorable de la intervención observando una ganancia de peso total durante la gestación inferior en el grupo de gestantes que recibieron intervención comparado con el control $(18,28,34,38,40,41)$. El estudio llevado a cabo por Hui A. y cols. (26), que expresa sus resultados en función del estado nutricional previo a la gestación, reporta una ganancia de peso total inferior en el subgrupo de embarazas con IMC normal que recibieron la intervención nutricional. Por su lado, el trabajo de Vitolo y cols., (47) que evalúa la ganancia de peso semanal, también observa una respuesta satisfactoria de la intervención en las mujeres que comenzaron su embarazo con exceso de peso.

$\mathrm{Al}$ analizar los resultados de las intervenciones según el grado de adherencia a las recomendaciones de ganancia de peso gestacional, cuatro estudios demostraron una menor proporción de embarazadas que se excedían en la ganancia de peso estipulada como objetivo en el grupo que recibió la intervención $(29,38,40,41)$. Sin embrago, algunos autores señalan la efectividad de la intervención en este sentido solamente en la sub muestra de mujeres con IMC normal previo a la gestación $(26,30-32)$. En el trabajo realizado por Olson y cols. (33), la intervención redujo significativamente el riesgo de una ganancia excesiva de peso exclusivamente en las gestantes de bajos recursos.

Dentro de los estudios orientados a abordar la malnutrición por déficit, las intervenciones educativas llevadas a cabo en India y Bangadesh con el propósito de estimular una ganancia ponderal adecuada demuestran resultados satisfactorios; Jahan y cols. (19) lograron este objetivo mediante la implementación de EAN durante el tercer trimestre de gestación y por su lado, Shivalli y cols. (17) también describen una ganancia de peso semanal mayor en el grupo de intervención. En América Latina, Restrepo S. y col. (46) también demuestran que luego de la aplicación de una intervención nutricional se registra una disminución de la prevalencia de bajo peso gestacional.

Tres de las investigaciones analizadas cuya finalidad fue lograr un efecto en la ganancia de peso durante la gestación no observaron diferencias significativas entre los grupos de intervención y control ya sea en la ganancia de peso total durante el embarazo o en la prevención de la ganancia excesiva de peso $(24,36,42)$. En la investigación de Althuizen y cols. 


\section{TABLA 3}

Estudios que tuvieron como objetivos lograr una adecuada ganancia de peso gestacional mediante intervenciones nutricionales (GI: grupo intervención; GC: grupo control)

Autor, Año, Referencias

Althuizen E. y cols. 2012 (42)

Asbee SM. y cols. 2009 (28)

Di Carlo C. y cols. 2014 (40)

Gesell SB. y cols. 2015 (30)

Huang T. y cols. 2011 (18)

Hui A. y cols. 2011 (29)

Hui A. y cols. 2014 (26)

Jackson R. y cols. 2011 (24)

Jahan K. y cols. 2014 (19)

Kinnunen T. y cols.2007 (36)

McGowan C. y cols. 2013 (38)

Olson C. M. y cols. 2004 (33)

Phelan S. y cols. 2011 (31)

Polley B. A. y cols. 2002 (32)

Rauh K. y cols. 2013 (41)

Restrepo S. y col. 2010 (46)

Ruchat S. y cols. 2012 (34)

Shivalli S. y cols. 2015 (17)

Vitolo M. R. y cols. 2011 (47)
País

Holanda

E.E.U.U.

Italia

E.E.U.U.

Taiwan

Canadá

Canadá

E.E.U.U.

Bangladesh

Finlandia

Irlanda

E.E.U.U.

E.E.U.U.

E.E.U.U.

Alemania

Colombia

Canadá

India

Brasil
Principales Hallazgos

La intervención no tuvo efecto sobre la ganancia de peso gestacional total $(11,6 \pm 4,1 \mathrm{~kg}$ vs 11,1 \pm $3,2 \mathrm{~kg}$ ) ni en la proporción de embarazadas que superaron la recomendación de ganancia de peso (70,4\% vs 72,4\%) entre los Gl y GC respectivamente.

Las participantes del $\mathrm{Gl}$ ganaron significativamente menos peso en comparación con las mujeres del GC $(13,2 \pm 5,67 \mathrm{~kg}$ vs 16,11 $\pm 7,03 \mathrm{~kg}$ respectivamente; $p=0,01)$. Sin embargo no hubo diferencias significativas entre ambos grupos en la tasa de adherencia a las recomendaciones de ganancia de peso ( $61,4 \%$ vs $48,8 \%$ adherentes en el GI y GC respectivamente; $p=0,21)$. El IMC pregestacional normal fue el mayor factor predictivo para la adherencia a las recomendaciones de ganancia de peso.

La ganancia de peso fue significativamente menor en el Gl comparado con el GC $(8,2 \pm 4,0 \mathrm{~kg}$ vs $13,4 \pm 4,2 \mathrm{~kg} ; \mathrm{p}<0,001)$. Asumiendo como saludable una ganancia de peso de hasta $12 \mathrm{~kg}$ en mujeres con IMC pregestacional normal, el $95,1 \%$ del $\mathrm{Gl}$ y el $41 \%$ del GC tuvo el incremento de peso esperado $(p<0,001)$.

No hubo diferencias significativas en el porcentaje de mujeres que excedieron las recomendaciones de ganancia de peso entre ambos grupos. En la subcategoría de peso normal pregestacional, la intervención registró una menor proporción de mujeres que excedieron las recomendaciones de ganancia de peso gestacional $(6,7 \%$ vs $47,1 \%$, Gl y GC respectivamente; $p=0,036)$. La ganancia de peso total gestacional no difirió entre ambos grupos $(8,85 \pm 5,57 \mathrm{~kg}$ vs $10,17 \pm 7,06 \mathrm{~kg} \mathrm{Gl} \mathrm{y} \mathrm{GCl}$ respectivamente; $p=0,374)$.

La ganancia de peso gestacional fue menor en el Gl comparada con el GC $(p<0,001)$.

La ganancia de peso gestacional no difirió entre los GI y GC $(14,1 \pm 6,0 \mathrm{~kg}$ vs 15,2 $\pm 5,9 \mathrm{~kg}$ respectivamente, $p=0,28$ ). La intervención redujo significativamente la prevalencia de aumento excesivo de peso según las recomendaciones en el GI vs GC (35,3\% vs 54,5\% respectivamente; $p=0,008)$

En la subcategoría de embarazadas con IMC pregestacional normal la intervención produjo una ganancia de peso gestacional menor $(12,9 \pm 3,72 \mathrm{~kg}$ vs $16,23 \pm 4,38 \mathrm{~kg}$; $p<0,05)$ y una tasa de ganancia de peso excesiva menor ( $10 \%$ versus $37 \%, p<0,05)$ comparada con el GC.

No hubo diferencias significativas en la ganancia de peso gestacional entre los grupos (15,15 kg en el Gl vs 15,24 kg en el GC; $p=0,95)$ ni entre la proporción de mujeres que excedieron la ganancia de peso recomendada.

En el Gl, el aumento de peso materno durante el tercer trimestre fue un $60 \%$ mayor ( $8,60 \mathrm{~kg}$ vs $5,38 \mathrm{~kg}, \mathrm{p}=0,011$ ) en comparación con el GC.

No hubo diferencias estadísticamente significativas en la ganancia de peso gestacional entre los $\mathrm{Gl}$ y GC $(14,6 \mathrm{~kg}$ vs $14,3 \mathrm{~kg}$ respectivamente; $p=0,77)$ ni entre la proporción de mujeres que excedieron las recomendaciones de ganancia de peso ( $46 \%$ vs $30 \%$, respectivamente).

La ganancia de peso gestacional fue significativamente menor en el Gl $(11,5 \mathrm{~kg}$ vs $12,6 \mathrm{~kg}$ para el Gl y GC respectivamente, $p=0,003)$. La ganancia de peso excesiva fue menor en el $\mathrm{Gl}$ comparado con el GC $(33,2 \%$ vs $44,7 \%$ respectivamente, $p=0,009)$.

La ganancia de peso total gestacional no difirió entre el Gl y el GC $(14,10 \pm 4,51 \mathrm{~kg}$ vs $14,80 \pm$ $4,68 \mathrm{~kg} ; \mathrm{p}=0,09)$, así como tampoco la proporción de mujeres que excedieron las recomendaciones de ganancia de peso ( $41 \%$ vs $45 \%$; $p=0,3$ ) respectivamente. Sin embargo, en las mujeres con bajo nivel de ingresos del $\mathrm{Gl}$, la proporción de ganancia de peso excesiva difirió de la del GC (33\% vs $52 \% ; p<0,01)$

Las mujeres con peso normal del GI tuvieron menos probabilidad de superar las recomendaciones de ganancia de peso (OR: 0,$38 ; 95 \% \mathrm{Cl}: 0,20-0,87 ; \mathrm{p}=0,003$ ), aunque este efecto no fue similar en la mujeres con exceso de peso (OR: 1,4; $95 \% \mathrm{Cl}: 0,70-2,7 ; \mathrm{p}=0,33$ ).

La intervención no tuvo efectos significativos sobre la ganancia de peso total gestacional. El porcentaje de mujeres de peso normal que excedían las recomendaciones de ganancia de peso fue menor en el Gl comparado con el GC ( $33 \%$ vs $58 \%, p<0,05)$. Entre las mujeres con sobrepeso no hubo diferencias significativas ( $59 \%$ Gl y $32 \%$ GC ganaron más peso que el recomendado; $p=0,09$ ). La ganancia de peso gestacional fue menor en el Gl comparado con el GC $(14,1 \mathrm{~kg} \pm 4,1 \mathrm{vs} 15,6 \mathrm{~kg}$ $\pm 5,8 ; p=0,049)$. La intervención resultó en una menor proporción de mujeres que superaron las recomendaciones de ganancia de peso ( $38 \%$ vs $60 \% ; p=0,032$ ).

El bajo peso gestacional disminuyó de $27,8 \%$ en el primer trimestre a $20,3 \%$ en el tercero. La ganancia de peso gestacional fue similar entre los dos $\mathrm{Gl}(15,3 \pm 2,9 \mathrm{~kg}$ y $14,9 \pm 3,8, \mathrm{p}=0,72)$ y menor que el GC $(18,3 \pm 5,3 \mathrm{~kg})$. En el GC la proporción de mujeres que ganan peso según lo recomendado fue del $47 \%$ y en los Gl $65 \%$ y $69 \%$, $p=0,32$.

La ganancia de peso semanal fue significativamente mayor en el Gl que en el GC $(326,9 \pm 91.8 \mathrm{~g}$ vs $244,6 \pm 97,4 \mathrm{~g}$; $p<0,01$ ).

La intervención fue efectiva en la reducción de la tasa de aumento de peso semanal de las mujeres embarazadas con exceso de peso $(342,2 \pm 143,6 \mathrm{~g}$ Gl vs 420,2 $\pm 185,4 \mathrm{~g} \mathrm{GC} ; \mathrm{p}=0,01)$. 
(42) Ilevada a cabo en Holanda, la ganancia de peso total no pudo ser evaluada ya que la medición se realizó entre las semanas 15 y 35 de gestación, por lo que los autores señalan este hecho como una debilidad metodológica del trabajo. En el caso de Jackson R. A. y cols., la aplicación de una intervención educativa basada en un video logró cambios favorables en otras variables como los hábitos dietéticos pero no en la ganancia de peso, las mujeres que fueron incluidas en la intervención tenían un promedio de 19 semanas de gestación, y varias ya habían superado la ganancia optima de peso, motivo que probablemente condicionó los resultados (24). En cuanto a la experiencia reportada por Kinnunen y cols. (36) la misma se basó en una intervención educativa personalizada destinada a gestantes de Finlandia que se implementó dese el inicio del embarazo, sin embargo las mujeres del grupo de intervención tenían al inicio del estudio un IMC superior a las del grupo control y por lo tanto un mayor riesgo de presentar una ganancia de peso excesiva.
Efectividad del uso de suplementos en conjunto con actividades educativas para la prevención de anemia.

La anemia, tanto por su magnitud como por sus múltiples consecuencias, constituye en la actualidad un problema prioritario de salud pública, por lo que la suplementación con hierro durante el embarazo es habitualmente una intervención rutinaria. Las 13 investigaciones analizadas en esta revisión que tuvieron como propósito evaluar una intervención nutricional con un componente educativo con el fin de prevenir la anemia ferropénica provienen de Asia y América Latina, en la Tabla 4 se presenta un resumen de las mismas. En todas se indicó la administración de suplementos de hierro, algunos trabajos incluyeron otros nutrientes, siendo las combinaciones más frecuentes hierro y ácido fólico $(17,20,48)$, hierro, ácido fólico y vitamina $C(21,45,46)$ y la administración de hierro, vitamina B12 y Zinc (14).

En aquellos trabajos que informaron la dosis de hierro suministrada con el suplemento, la cantidad del mismo estuvo

\section{TABLA 4}

Estudios que tuvieron como objetivos prevenir la anemia durante el embarazo mediante intervenciones nutricionales focalizadas en la suplementación y la educación alimentaria (Gl: grupo intervención; GC: grupo control, Hb: Hemoglobina).

\begin{tabular}{ll}
\hline Autor, Año, Referencias & País \\
Adhikari K. y cols. 2009 (22) & Nepal
\end{tabular}

Adhikari K. y cols. 2009 (22)

Nepal

Aikawa R. y cols. 2008 (20)

Vietnam

Al-Tell MA. y cols. 2010 (15)

Palestina

Barbosa Chagas C. y cols. 2011 (48)

Brasil

Garg A. y cols. 2006 (16)

India

Khorshid MR. y cols. 2014 (23)

Irán

Ndiaye M. y cols. 2009 (53)

Senegal

Noronha J. y cols. 2013 (14)

India

Parra B. E. y col. 2005 (45)

Colombia

Restrepo S. y col. 2010 (46)

Senanayake HM. y cols. 2010 (21)

Shivalli S. y cols. 2015 (17)

India

Colombia

Sri Lanka

Vitolo M. R. y cols. 2006 (49)
Principales Hallazgos

El grupo que recibió solo educación y el grupo de conteo de píldoras más educación, incrementó significativamente los niveles de $\mathrm{Hb}$ en comparación con el GC $(p<0,01)$. La anemia fue reducida en un 59\% en el grupo de educación y en un $65 \%$ en el grupo de conteo de píldoras con educación, en comparación con el GC ( $p<0,05)$.

La suplementación con hierro incrementó significativamente la concentración de $\mathrm{Hb}$ entre las participantes beneficiarios del programa en el segundo y tercer trimestre $(p=0,017$ y $p<0,001$ respectivamente). El riesgo de anemia se disminuyó significativamente al tomar el suplemento $(p=0,041)$.

Se observó una toma irregular del suplemento en ambos grupos (58,8\% Gl y 54,9\% GC). El 13,7\% del Gl y el 19,6\% del GC refirieron no tomar el suplemento. La prevalencia de anemia disminuyo significativamente entre ambos grupos en el segundo y tercer trimestre de embarazo $(p<0,001)$ La intervención demostró tener un efecto protector sobre la anemia $(O R=0,420,95 \% \mathrm{Cl}=0,25$ $0,70)$ y una reducción significativa en su prevalencia $(28,4 \%$ vs $16,8 \%, p=0,001)$.

La consejería nutricional incremento los niveles medio de $\mathrm{Hb}$ del $\mathrm{Gl}$ comparado con el GC $(9,65 \pm$ $0,97$ vs 7,85 $\pm 1,58 ; p<0,001)$, así como disminuyo la prevalencia de anemia (78.7\% vs $96 \%)$. Hubo una mejoría en la adherencia a la toma del suplemento en el Gl.

Las mujeres del GI tuvieron una mayor adherencia a la toma del suplemento en comparación con el GC (94\% vs 66\%; $p=0,003)$. No hubo diferencias significativas entre los grupos con respecto al nivel de $\mathrm{Hb}$, hematocrito y ferritina sérica.

En el $\mathrm{Gl}$ el promedio de $\mathrm{Hb}$ se incrementó significativamente antes y después de la intervención (de 93,9 g/L a 100,7 g/L, p=0,011). La intervención redujo significativamente la prevalencia de anemia ( $55 \%$ vs $66 \%, p=0,003$ ) y mejoro la adherencia a la toma del suplemento ( $43 \%$ vs $26 \%$, $p=0,001)$.

El 61,2\% de las participantes en el Gl ya no presentaban anemia después de la intervención comparada con el $46,77 \%$ de GC $(p<0,01)$. El nivel de Hb se incrementó en el $\mathrm{Gl}$ comparada con el GC ( $p<0,001)$.

El $94 \%$ de las participantes no presentó anemia al final de la gestación y un 77,8\% mantuvieron los depósitos de hierro. Todas las madres ingirieron en promedio el 91\% de las tabletas y el 95\% de las madres toleraron el suplemento.

La anemia se previno en el $86 \%$ de las madres con deficiencia de hierro.

$\mathrm{La}$ intervención produjo una mejoría en el nivel de $\mathrm{Hb}$ plasmática (12 g/dL vs 11,1 g/dL; $\mathrm{p}<0.001)$, en la prevalencia de anemia (14\% vs 46,8\%; $p<0,001)$ y en el nivel de ferritina sérica $(28,6 \mathrm{ng} / \mathrm{L}$ vs $18,7$ ng/L; $p<0,001)$ entre el Gl y GC en la semana 34 de gestación. Así mismo el GI mejoró la biodisponibilidad del suplemento.

El nivel de $\mathrm{Hb}$ plasmática aumentó en el GI comparado con el GC $(p<0,001)$ La prevalencia de anemia se redujo a la mitad en el GI y un $2,4 \%$ en el GC $p<0,001)$. Más del $85 \%$ de las mujeres el $\mathrm{Gl}$ adhirió al suplemento mientras que solo el $38 \%$ del GC $(p<0,05)$.

La prevalencia de anemia al final del tercer trimestre fue de $31,6 \%$ en el GI y de $26,1 \%$ en el GC $(p=0,43)$. El uso del suplemento se informó en un $65 \%$ de las mujeres en el Gl, de los cuales $67,7 \%$ interrumpió el tratamiento en algún momento. 
comprendida entre 30 y $100 \mathrm{mg} /$ día de hierro elemental (14, $17,20-23,45,46,48,49)$, destacándose en siete de ellos el uso de 60 mg/día (20-22, 45, 46, 48, 49). Aquellos autores que adicionaron ácido fólico lo hicieron en el orden de los 400 a 500 mg/día $(17,20,45,46)$, a excepción del trabajo de Barbosa-Chagas y cols. que utilizaron $5 \mathrm{mg} /$ día de ácido fólico (48) y los dos que informaron la cantidad de vitamina C administraron $70 \mathrm{mg} /$ día $(45,46)$.

La suplementación con hierro cuando es administrada en cantidad y tiempos adecuados junto con educación para la salud logra demostrar o bien incrementos en los niveles de hemoglobina post intervención, o resultados netamente positivos en disminuir la prevalencia de anemia o el riesgo de padecerla $(14-17,20-22,48,49,53)$.

El grado de adherencia al uso de suplementos es probablemente uno de los condicionantes que influye en estos resultados favorables y los estudios demuestran que esta adherencia es significativamente mayor cuando está presente el componente educativo $(16,17,21,23,45,53)$.

\section{CONCLUSIONES}

El análisis de las experiencias de intervención nutricional destinadas a gestantes sin patologías provenientes de diferentes contextos geográficos, culturales y socioeconómicos que se incluyeron en esta revisión revelan resultados favorables especialmente cuando las mismas están destinadas a lograr un incremento en el nivel de conocimientos, a estimular cambios en los patrones dietéticos o a prevenir la anemia ferropénica mediante la adherencia a la suplementación con hierro.

Un aspecto controversial que merece ser profundizado en relación a los cambios en los hábitos alimentarios generados a partir de intervenciones educativas durante el embarazo, es conocer en qué medida cuando se logran modificaciones, las mismas son sostenidas durante el periodo de lactancia. La percepción de riesgo que la mujer reconoce durante el periodo de embarazo disminuye luego del nacimiento y por lo tanto las conductas de autocuidado, entre ellas los hábitos alimentarios pueden ser consideradas menos relevantes.

La ganancia de peso es también un indicador que se ve afectado favorablemente cuando se incluyen instancias de consejería nutricional a lo largo del embarazo, no obstante el prevenir ganancias excesivas depende del estado nutricional previo a la gestación y de otros condicionantes genéticos y ambientales siendo por lo tanto más compleja su evaluación.

El impacto de una intervención educativa es el resultado de múltiples factores entre los cuales deben tenerse en cuenta su adaptación a la población objetivo, la modalidad didáctica seleccionada y su frecuencia de administración. Otros aspectos metodológicos como la selección de un grupo control de iguales características al grupo de intervención, un adecuado tamaño muestral y la aleatoridad en la selección de los beneficiarios deben ser tenidos en cuenta a la hora de evaluar los resultados obtenidos.

En concordancia con nuestras apreciaciones, otros estudios de revisión han reportado efectos beneficiosos asociados a la EAN durante la gestación, no obstante varios autores destacan que aun es escaso el número de estudios que cumplan con los criterios metodológicos apropiados (59-64), por lo que promueven la generación de nuevas investigaciones en el tema. Sin embargo, las experiencias exitosas provenientes de diversos ámbitos geográficos estimulan la inclusión de un componente educativo con carácter rutinario en el control prenatal, la consejería nutricional personalizada así como el uso de Tics probablemente sean las alternativas de elección en el actual escenario sanitario.

\section{RESUMEN}

El embarazo es un momento de vulnerabilidad nutricional, por lo que la Educación Alimentaria Nutricional (EAN) resulta una herramienta valiosa para alcanzar una alimentación saludable. El objetivo de este trabajo es realizar una revisión de los impactos que tuvieron experiencias de EAN en mujeres embarazadas adultas sanas en distintos ámbitos geográficos. Se incluyeron 42 investigaciones publicadas entre los años 2000 y 2015. Se describió la procedencia de las publicaciones y las estrategias educativas empleadas. Se analizaron los efectos en los conocimientos, actitudes y prácticas alimentarias, en el patrón de ganancia de peso y en estimular la adherencia a la suplementación con hierro. Predominaron las investigaciones de Asia y las que incluyeron una combinación de múltiples estrategias educativas. Las intervenciones revelan resultados favorables cuando se proponen mejorar el nivel de conocimientos, estimular cambios en los patrones dietéticos y prevenir la anemia ferropénica mediante suplementación. La ganancia de peso se afectó favorablemente, no obstante el prevenir ganancias excesivas depende del estado nutricional pre-gestacional y de otros condicionantes genéticos y ambientales. Las experiencias exitosas estimulan la inclusión de EAN rutinaria en el control prenatal, siendo la consejería nutricional personalizada y el uso de Tics las alternativas de elección.

Palabras clave: Embarazo; nutrición durante el embarazo; control prenatal; educación para la salud; intervención nutricional.

Agradecimientos. La investigación fue financiada por la Universidad de Buenos Aires mediante la Programación Científica y Técnica UBACYT 2012-2015 (Código: 20020110100181) y el Proyecto de Desarrollo Tecnológico y Social (Código: M03).

Conflicto de interés. Los autores declaran que no existen conflictos de interés relacionados con el manuscrito.

\section{BIBLIOGRAFIA}

1. Mehta SH. Nutrition and pregnancy. Clin Obstet Gynecol. 2008; 51(2):409-18.

2. King JC. Physiology of pregnancy and nutrient metabolism. Am J Clin Nutr. 2000; 71(suppl):1218-25.

3. Christian P, Mullany LC, Hurley KM, Katz J, Black RE. Nutrition and maternal, neonatal, and child health. Semin Perinatol. 2015; 39(5):361-72.

4. Food and Agriculture Organization of the United. Nutrition education for the public. Discussion papers of the FAO Expert Consultation (Rome, Italy 18-22 September 1995) - FAO Food and Nutrition Paper 62.

5. Leddy M, Power M, Schulkin J. The Impact of Maternal Obesity on Maternal and Fetal Health. Rev Obstet Gynecol. 2008; 1(4):170-8.

6. Oteng-Ntim E, Varma $R$, Croker $H$, Poston L, Doyle P. Lifestyle interventions for overweight and obese pregnant women to improve pregnancy outcome: systematic review and meta-analysis. BMC Med. 2012;10:47

7. Triunfo $S$, Lanzone $A$. Impact of maternal under nutrition on obstetric outcomes, J Endocrinol Invest. 2015; 38(1):31-8.

8. Ramírez-Vélez $R$. In Utero Fetal Programming and Its Impact on Health in Adulthood. Endocrinol Nutr. 2012; 59(6):383-93. 
9. World Health Organization. Global prevalence of anaemia in 2011. Geneva, 2015.

10. World Health Organization. Essential Nutrition Actions: improving maternal, newborn, infant and young child health and nutrition. Geneva, 2013.

11. Phelan S. Pregnancy: a "teachable moment" for weight control and obesity prevention. Am J Obstet Gynecol. 2010; 202:135.e1-8.

12. Fallah F, Pourabbas A, Delpisheh A, Veisani $Y$, Shadnoush $M$. Effects of nutrition educationon levels of nutritional awareness of pregnant women in Western Iran. Int J Endocrinol Metab. 2013; 11(3):175-8.

13. Khoramabadi M, Dolatian M, Hajian S, Zamanian M, Taheripanah R, Sheikhan Z. y cols. Effects of Education Based on Health Belief Model on Dietary Behaviors of Iranian Pregnant Women. Glob J Health Sci. 201; 25;8(2):230-9.

14. Noronha JA, Bhaduri A, Vinod Bha H, Kamath A. Interventional study to strengthen the health promoting behaviours of pregnant women to prevent anaemia in southern India. Midwifery. 2013; 29(7):35-41

15. Al-Tell MA, El-Guindi FK, Soliman NM, El-Nana H. Effect of Nutritional Interventions on Anemic Pregnant Women's Health Using Health Promotion Model. Med. J. Cairo Univ. 2010; 78(2):109-18.

16. Garg A, Kashyap S. Effect of counseling on nutritional status during pregnancy. Indian J Pediatr. 2006; 73(8):68792.

17. Shivalli S, Srivastava RK, Singh GP. Trials of Improved Practices (TIPS) to Enhance the Dietary and Iron-Folate Intake during Pregnancy- A Quasi Experimental Study among Rural Pregnant Women of Varanasi, India. PLoS One. 2015; 14; 10(9):e0137735

18. Huang T, Yeh C, Tsai Y. A diet and physical activity intervention for preventing weight retention among Taiwanese childbearing women: a randomised controlled trial. Midwifery. 2011; 27(2):257-64.

19. Jahan K, Roy SK, Mihrshahi S, Sultana N, Khatoon S, Roy $\mathrm{H}$ y cols. Short-term nutrition education reduces low birthweight and improves pregnancy outcomes among urban poor women in Bangladesh. Food Nutr Bull. 2014; 35(4):414-21.

20. Aikawa $R$, Jimba M, Nguen KC, Binns CW. Prenatal iron supplementation in rural Vietnam. Eur J Clin Nutr. 2008; 62(8):946-52.

21. Senanayake HM, Premaratne SP, Palihawadana T, Wijeratne S. Simple educational intervention will improve the efficacy of routine antenatal iron supplementation. J Obstet Gynaecol Res. 2010; 36(3):646-50.

22. Adhikari K, Liabsuetrakul T Pradhan N. Effect of education and pill count on hemoglobin status during prenatal care in Nepalese women: A randomized controlled trial. J Obstet Gynaecol Res. 2009; 5(3):459-66.

23. Khorshid MR, Afshari P, Abedi P. The effect of SMS messaging on the compliance with iron supplementation among pregnant women in Iran: a randomized controlled trial. J Telemed Telecare.2014; 20(4):201-6

24. Jackson RA, Stotland NE, Caughey AB, Gerbert B. Improving diet and exercise in pregnancy with Video Doctor counseling: a randomized trial. Patient Educ Couns. 2011; 83(2):203-9.

25. Briley C, Flanagan NL, Lewis NM. In-home Prenatal Nutrition Intervention Increased Dietary Iron Intakes and Reduced Low Birthweight in Low-Income African-American Women. J Am Diet Assoc. 2002; 102(7):984-7.
26. Hui AL, Back L, Ludwig S, Gardiner P, Sevenhuysen $G$, Dean H. y cols. Effects of lifestyle intervention on dietary intake, physical activity level, and gestational weight gain in pregnant women with different pre-pregnancy Body Mass Index in a randomized control trial. BMC Pregnancy Childbirth. 2014; 14:331

27. Oken E, Guthrie LB, Bloomingdale A, Platek DN, Price $S$, Haines J. y cols. A pilot randomized controlled trial to promote healthful fish consumption during pregnancy: The Food for Thought Study. Nutr J. 2013; Mar 15;12:33.

28. Asbee SM, Jenkins TR, Butler JR, White J, Elliot M, Rutledge A. Preventing Excessive Weight Gain During Pregnancy Through Dietary and Lifestyle Counseling. Obstet Gynecol. 2009; 113(2):305-12.

29. Hui A, Back L, Ludwig S, Gardiner P, Sevenhuysen G, Dean $H$. y cols. Lifestyle intervention on diet and exercise reduced excessive gestational weight gain in pregnant women under a randomised controlled trial. BJOG. 2011; 119(1):70-77.

30. Gesell SB, Katula JA, Strickland C, Vitolins MZ. Feasibility and Initial Efficacy Evaluation of a Community-Based Cognitive-Behavioral Lifestyle Intervention to Prevent Excessive Weight Gain During Pregnancy in Latina Women. Matern Child Health J. 2015; 19:1842-52.

31. Phelan S, Phipps MG, Abrams B, Darroch F, Schaffner A, Wing RR. Randomized trial of a behavioral intervention to prevent excessive gestational weight gain: the Fit for Delivery Study. Am J Clin Nutr. 2011; 93(4):772-9.

32. Polley BA, Wing RR, Sims CJ. Randomized controlled trial to prevent excessive weight gain in pregnant women. Int J Obes. 2002;26(11):1494-502.

33. Olson CM, Strawderman MS, Reed RG. Efficacy of an intervention to prevent excessive gestational weight gain. Am J Obstet Gynecol. 2004; 191(2):530-6.

34. Ruchat S, Davenport MH, Giroux I, Hillier M, Batada A, Sopper M. y cols. Nutrition and Exercise Reduce Excessive Weight Gain in Normal-Weight Pregnant Women. Med Sci Sports Exerc. 2012; 44(8):1419-26.

35. Bosaeus M, Hussain A, Karlsson T, Andersson L, Hulthén $L$, Svelander $C$, y cols. A randomized longitudinal dietary intervention study during pregnancy: effects on fish intake, phospholipids, and body composition. Nutr J. 2015; Jan 2;14:1.

36. Kinnunen TI, Pasanen M, Aittasalo M, Fogelholm M, Hilakivi-Clarke L, Weiderpass $E$, y cols. Preventing excessive weight gain during pregnancy: a controlled trial in primary health care. Eur J Clin Nutr. 2007; 61(7):884-91.

37. Laitinen $K$, Poussa $T$, Isolauri E. Probiotics and dietary counselling contribute to glucose regulation during and after pregnancy: a randomized controlled trial. Br J Nutr. 2009; 101(11):1679-87.

38. McGowan CA, Walsh JM, Byrne J, Curran S, McAuliffe FA. The influence of a low glycemic index dietary intervention on maternal dietary intake, glycemic index and gestational weight gain during pregnancy: a randomized controlled trial. Nutr J. 2013; 12(1):140.

39. Piirainen $T$, Isolauri $E$, Lagstrom $H$, Laitinen $K$. Impact of dietary counselling on nutrient intake during pregnancy: a prospective cohort study. Br J Nutr. 2006; 96(06):1095104.

40. Di Carlo C, lannotti G, Sparice S, Chiacchio MP, Greco E, Tommaselli $G, y$ cols. The role of a personalized dietary intervention in managing gestational weight gain: a prospective, controlled study in a low-risk antenatal population. Arch Gynecol Obstet. 2014; 289(4):765-70. 
41. Rauh K, Gabriel E, Kerschbaum E, Schuster $T$, Kries $R$, Amann-Gassner $U, y$ cols. Safety and efficacy of a lifestyle intervention for pregnant women to prevent excessive maternal weight gain: a cluster-randomized controlled trial. BMC Pregnancy Childbirth. 2013; 13:151.

42. Althuizen $E$, van der Wijden $C L$, van Mechelen $W$, Seidell $J C$, van Poppel MNM. The effect of a counselling intervention on weight changes during and after pregnancy: a randomised trial. BJOG. 2012; 120(1):92-9.

43. Gutierrez-Maydata A, Treto-Fernandez M, Wong Orfila T, González Ramirez JA. Use of national educational television in a local project to increase the consumption of antioxidant vitamins among pregnant women Rev Cubana Obstet Ginecol. 2006; 32(2):1-9.

44. Moreno Mejia C, Rivera Tejada HS. Influence of an educational program food on the level knowledge center on the pregnant health Los Granados-Trujillo. In Crescendo. 2011; 2(1):55-67

45. Parra $B E$, Manjarrés $L M$, Gómez $A L$, Alzate DM, Jaramillo MC. Assessment of nutritional education and iron supplement impact on prevention of pregnancy anemia. Biomédica. 2005; 25:211-9

46. Restrepo SL, Mancilla LP, Parra BE, Manjarrés LM, Zapata NJ, Restrepo Ochoa PA, y col. Assessment of nutritional status of a group of pregnant women who participated in a food and nutrition program. Rev Chil Nutr. 2010; 37(1):18-30

47. Vitolo MR., Fraga Bueno MS, Mendes Gama C. Impact of a dietary counseling program on the gain weight speed of pregnant women attended in a primary care service. Rev Bras Ginecol Obstet. 2011; 33(1):13-9.

48. Barbosa Chagas $C$, Ramalho A, Padilha P, Della Libera $B$, Saunders $C$. Reduction of vitamin $A$ deficiency and anemia in pregnancy after implementing proposed prenatal nutritional assistance. Nutr Hosp. 2011; 26(4):843-50.

49. Vitolo MR. Boscaini C, Bortolini GA. Low educational level as a limiting factor in the fight against anemia in pregnant women. Rev Bras Ginecol Obstet. 2006;28(6): 331-9.

50. Emmett $R$, Akkersdyk S, Yeatman H, Meyer BJ. Expanding awareness of docosahexaenoic acid during pregnancy. Nutrients. 2013; 5(4):1098-109.

51. Wilkinson SA, Mclntyre HD. Evaluation of the healthy start to pregnancy early antenatal health promotion workshop: a randomized controlled trial. BMC Pregnancy Childbirth. 2012; 12(1):131.

52. Ahrari M, Houser RF, Yassin S, Mogheez M, Hussaini $Y$, Crump P, y cols. A positive deviance-based antenatal nutrition project improves birth-weight in Upper Egypt. J Health Popul Nutr. 2006; 24(4):498-507
53. Ndiaye $M$, Siekmans $K$, Haddad S, Receveur O. Impact of a positive deviance approach to improve the effectiveness of an iron-supplementation program to control nutritional anemia among rural Senegalese pregnant women. Food Nutr Bull. 2009; Jun; 30(2):128-36.

54. World Health Organization and International Telecommunication Union. eHealth and innovation in women's and children's health: a baseline review: based on the findings of the 2013 survey of ColA countries by the WHO Global Observatory for eHealth; 2014: 1-164.

55. World Health Organization. Building Foundations for eHealth in Europe. Report of the WHO Global Observatory for eHealth. 2008.

56. Latham MC. Human nutrition in the developing world. Food and Nutrition Series - N²9. 1997.

57. Institute of Medicine Committee on Nutritional Status During Pregnancy and Lactation. Nutrition During Pregnancy: Part I Weight Gain: Part II Nutrient Supplements. Washington (DC): National Academies Press (US); 1990.

58. Institute of Medicine and National Research Committee to Reexamine IOM Pregnancy Weight Guidelines. Weight Gain During Pregnancy: Reexamining the Guidelines. Washington, DC: National Academies Press; 2009.

59. Girard AW, Olude O. Nutrition Education and Counselling Provided during Pregnancy: Effects on Maternal, Neonatal and Child Health Outcomes. Paediatr Perinat Epidemiol. 2012; 26:191-204.

60. Muktabhant B, Lawrie TA, Lumbiganon P, Laopaiboon M. Diet or exercise, or both, for preventing excessive weight gain in pregnancy. Cochrane Database Syst Rev. 2015; 6:CD007145

61. O'Brien OA, McCarthy M, Gibney ER, McAuliffe FM. Technology-supported dietary and lifestyle interventions in healthy pregnant women: a systematic review. Eur J Clin Nutr. 2014; 68(7):760-6.

62. O'Brien CM, Grivell RM, Dodd JM. Systematic review of antenatal dietary and lifestyle interventions in women with a normal body mass index. Acta Obstet Gynecol Scand. 2016; 95(3):259-69.

63. Tanentsapf I, Heitmann BL, Adegboye AR. Systematic review of clinical trials on dietary interventions to prevent excessive weight gain during pregnancy among normal weight, overweight and obese women. BMC Pregnancy Childbirth. 2011; 11:81

64. Thangaratinam S, Rogozinska E, Jolly K, Glinkowski S, Roseboom T, Tomlinson JW, Kunz R, Mol BW, Coomarasamy $A$, Khan $K S$. Effects of interventions in pregnancy on maternal weight and obstetric outcomes: meta-analysis of randomised evidence. BMJ. 2012 May 16;344:e2088. 